\title{
Memory Traces of Pain in Human Cortex
}

\author{
Marie-Claire Albanese, ${ }^{1}$ Emma G. Duerden, ${ }^{3}$ Pierre Rainville, ${ }^{3,4}$ and Gary H. Duncan ${ }^{2,3,4}$ \\ Departments of ${ }^{1}$ Psychology and ${ }^{2}$ Neurology and Neurosurgery, McGill University, Montréal, Québec, Canada H3A 1B1, and ${ }^{3} \mathrm{Groupe}$ de recherche sur le \\ système nerveux central and ${ }^{4}$ Département de stomatologie, Université de Montréal, Montréal, Québec, Canada H3T 1J4
}

Distinct brain regions process sensory discriminative and affective components of pain; however, the role of these areas in pain memory is unknown. This event-related study investigated the short-term memory for sensory features of cutaneous heat pain using a delayeddiscrimination paradigm and functional magnetic resonance imaging. During memory trials, subjects discriminated the location and intensity of two painful stimuli presented sequentially to the right hand. Control trials comprised the same sequence of stimuli and motor responses but required no delayed discrimination. Stimulus-evoked activity for memory and control trials was generally indistinguishable within the network of regions normally responsive to experimental pain [i.e., the primary somatosensory cortex/posterior parietal cortex (SI/PPC), secondary somatosensory cortex (SII), and anterior insular cortex (aIC)]; these data confirm the painful nature of the stimuli and the similar levels of attention and stimulus encoding engaged during the two randomly presented trial types. Memoryspecific activity, assessed by contrasting the interstimulus interval in memory and control trials, was observed in SI/PPC and aIC but not in SII. We propose that SI/PPC plays a role in the short-term retention of spatial and intensity aspects of noxious stimuli and that aIC activation during memory trials is consistent with the integration of sensory and cognitive (attention, awareness, salience, and memory) components of pain perception. The absence of memory-specific anterior cingulate cortex activation, generally associated with pain unpleasantness, suggests that remembering affective aspects of the stimuli was not required during performance of the sensory delayeddiscrimination task.

Key words: pain; sensory discrimination; working memory; fMRI; somatosensory cortex; prefrontal cortex

\section{Introduction}

The remembered experience of pain corresponds to a persisting mental representation of a previous exposure to noxious stimulation. As such, this pain memory is at the heart of a broad spectrum of theories that includes the shaping of subsequent perception and behavior (e.g., in pain anticipation) (Taddio et al., 1995; Porro et al., 2002; Koyama et al., 2005), recognition of pain in others (Singer et al., 2004), and the transformation of acute peripheral injury to centralized chronic pain (Woolf and Salter, 2000). Common to these theoretical perspectives is the notion that pain memory relies on neural networks involved in pain experiences that are activated in the absence of the physical application of a noxious stimulus.

The conscious experience of pain is a consequence of complex interactions among distinct brain regions, which appear to preferentially process sensory-discriminative and affective aspects of the noxious stimulation. Previous studies examining painrelated activation in the brain have consistently found that, under normal conditions, several cortical structures, including primary and secondary somatosensory areas (SI and SII), the insular cortex (IC), and the anterior cingulate cortex (ACC), display levels of

Received June 15, 2006; revised March 16, 2007; accepted March 19, 2007.

This work was supported by grants from the Canadian Institutes of Health Research and the National Sciences and Engineering Research Council of Canada. We thank M. Catherine Bushnell and Michael Petrides for comments on a previous version of this manuscript and Leo Ten Bokum for technical assistance.

Correspondence should be addressed to Dr. Gary H. Duncan, 2960 Chemin de la Tour, Room 4135, Université de Montréal, Montréal, Québec, Canada H3T 1J4. E-mail: gary.duncan@umontreal.ca.

DOl:10.1523/JNEUROSCI.0695-07.2007

Copyright $\odot 2007$ Society for Neuroscience $\quad$ 0270-6474/07/274612-09\$15.00/0 activation that parallel the intensity of the stimulus and the intensity of pain perceived (Porro et al., 1998; Coghill et al., 2001, 2003). In addition, we and others have shown that some of these areas, such as the ACC and possibly part of the insula, appear to be more strongly associated with the affective dimension of pain (Rainville et al., 1997; Coghill et al., 2003; Wager et al., 2004), findings that do not preclude a role for these structures in the coding of intensity, because the perception of pain intensity and affect are often highly correlated (Rainville et al., 1992). The role of these various brain areas in pain memory is still uncertain.

Human and animal studies have suggested that the short-term memory of nonpainful sensory information involves both prefrontal areas and cortical regions that subserve the initial sensory processing of those stimuli (Pasternak and Greenlee, 2005); however, no studies have yet explored the cerebral areas selectively involved in the explicit, conscious memory of the sensory aspects of pain. In contrast, memory of the affective aspect of pain has been proposed as a critical element in the expression of pain empathy (Preston and de Waal, 2002), an idea supported by recent studies showing that visual cues signaling pain in others or images representing painful situations most commonly activate the ACC (Morrison et al., 2004; Singer et al., 2004; Jackson et al., 2005). However, considering the highly correlated nature of pain intensity and pain affect, each of the structures showing painrelated activation, including SI, SII, the IC, and the ACC, is a potential candidate to contribute to the short-term memory for sensory features of a pain stimulus. In the present study, we specifically investigated the neural correlates of short-term memory 


\section{Stimulation Protocol}
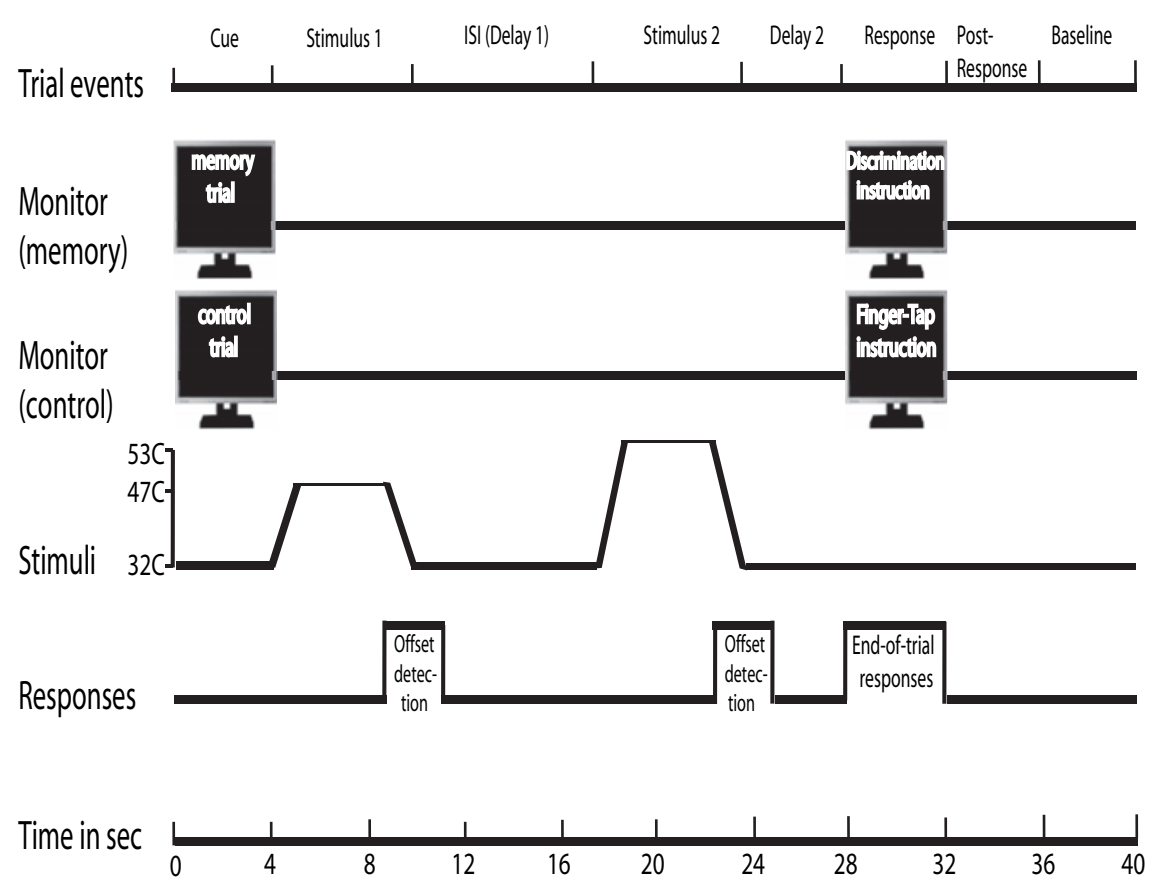

Figure 1. Schematic representation of the stimulation protocol with an $8 \mathrm{~s} \mid \mathrm{SI}$; the memory and control trials were presented in a pseudo-randomized order. See Materials and Methods, Experimental paradigm, for a description of each time period for both trial types.

for the sensory aspects of pain, focusing on spatial and intensity features of brief heat-pain stimuli.

\section{Materials and Methods}

Subjects. Eight right-handed normal volunteers (four females and four males; mean age, 34 years; SD, 9.3) were recruited for a pain and memory functional magnetic resonance imaging (fMRI) study. Before the scanning sessions, we established the pain threshold for each subject, and all subjects were familiarized with the stimuli and the experimental design. The Research Ethics Board of the Montreal Neurological Institute and Hospital approved the study. All subjects gave written informed consent and were financially compensated for their time commitment to this study.

Stimuli. Thermal stimuli $\left(47.5-53^{\circ} \mathrm{C}\right.$ for heat pain) were delivered by two contact thermodes (TSA Peltier device, $9 \mathrm{~cm}^{2}$; Medoc Advanced Medical Systems, Ramat Yishai, Israel) placed on the thenar and hypothenar eminences of the subjects' right hand. On each discrimination trial, the two stimuli were presented sequentially to the two stimulation sites, the order of which was counterbalanced and pseudo-randomized across trials. The differences in intensity $(\Delta T s)$ of the two stimulus presentations ranged from 0.1 to $1.5^{\circ} \mathrm{C}$ and were, likewise, counterbalanced and pseudo-randomized across trials. The magnitude of $\Delta T s$ was chosen to ensure task difficulty and minimize the subjects' tendency to categorize sensory characteristics of the noxious stimuli. Specific temperatures presented to individual subjects during individual runs were adapted to the perception of the subject, to maintain a (post-run) rating of moderate pain.

Experimental paradigm. The delayed-discrimination task (Fig. 1) evolved from a series of psychophysical tasks specifically designed to study the memory of pain sensation intensity (Rainville et al., 2004). At the beginning of each trial, subjects viewed a written cue on the monitor ( $4 \mathrm{~s}$ duration) indicating whether the following trial was a memory or a control trial ("memory trial" or "offset trial", respectively). After the cue, a first heat stimulus ( $6 \mathrm{~s}$ duration, $4 \mathrm{~s}$ plateau) was delivered to one of the two locations on the right hand, followed by an interstimulus interval
(ISI; delay 1 ) of 6,8 , or $10 \mathrm{~s}$ and a second stimulus ( $6 \mathrm{~s}$ duration, $4 \mathrm{~s}$ plateau) delivered to the other stimulation site. A second delay (delay 2) of $6 \mathrm{~s}$ separated the stimulation period from the response period. In memory trials, subjects were directed by a prompt on the monitor $(4 \mathrm{~s}$ duration) to indicate the location of the stronger (or weaker) stimulus ("which stimulus greater?" or "which stimulus less?"). These response prompts were presented in a pseudorandom manner (1:1) among memory trials to ensure that subjects could not prepare their specific sensorimotor decision and motor response before the end of delay 2 . Subjects responded by tapping on an fMRI-compatible mouse button (left hand: digit 2, thenar stimulation; digit 3, hypothenar stimulation) until the discrimination question disappeared from the screen. In the control trials, subjects were similarly instructed either to tap "digit 2" or "digit 3 " of the left hand on the appropriate mouse button for the $4 \mathrm{~s}$ response period. (This $4 \mathrm{~s}$ left hand tapping at the end of the trial was designed to produce a robust motor activation, which could be used as a standard of comparison for pain-related activation evoked by the noxious stimuli presented to the opposite hand during the stimulation period of the trial). Each trial ended with $4 \mathrm{~s}$ post-response and baseline periods, the latter being used as a basis for comparison during the analysis of stimulus- and memory-related activity.

The control trials were comparable to the memory trials with respect to visual and thermal stimuli, as well as motor responses, except that control trials did not require subjects to perform any sensory discrimination between the pairs of stimuli. To standardize and maximize attention to the entire duration of the stimulus presentations during both memory and control trials, subjects were required to detect the termination (offset) of each thermal stimulus and respond by producing a single tap of the mouse with their left hand.

$f M R I$ acquisition. Cerebral activity associated with perception, encoding, or retention of the heat-pain stimuli was measured with fMRI. We acquired the imaging data at the McConnell Brain Imaging Center of the Montreal Neurological Institute at McGill University on a 1.5T Siemens (Munich, Germany) Sonata scanner using a standard head coil. The MR technician fixed the head of each subject in a comfortable position and immobilized it with a vacuum bag. Subjects were instructed to refrain as much as possible from moving throughout the imaging session and were given earplugs to reduce the noise from the scanner. Subjects could see a computer monitor projected on a screen viewed via a mirror placed in front of the subject's eyes. Subjects were also given an MR-compatible mouse to perform the discrimination task. Each session consisted of an anatomical scan and three to six functional runs. The anatomical scans were T1-weighted high-resolution scans [repetition time (TR), $22 \mathrm{~ms}$; echo time (TE), $20 \mathrm{~ms}$; flip angle, $30^{\circ}$; field of view, $256 \mathrm{~mm} ; 1 \mathrm{~mm}$ isotropic sampling]. The functional scans were collected using a blood oxygen level-dependent (BOLD) protocol with a $\mathrm{T} 2^{\star}$-weighted gradient echo-planar imaging sequence (TR, $2.0 \mathrm{~s}$; TE, $51 \mathrm{~ms}$; flip angle, $90^{\circ}$; $64 \times$ 64 matrix; 253 volume acquisitions). The scanning planes were oriented parallel to the anterior commissure-posterior commissure line and covered part of the brain from the top of the cortex to the base of the thalamus ( 23 contiguous 5 -mm-thick slices; voxel size, $5 \times 5 \times 5 \mathrm{~mm}$ ).

Data analysis. Functional data and anatomical images were analyzed using Brain Voyager QX (Brain Innovation, Maastricht, The Netherlands). Functional images were preprocessed (interscan slice time correction, three-dimensional motion correction, high-pass filtering), in terpolated to $1 \times 1 \times 1 \mathrm{~mm}^{3}$, and coregistered to three-dimensional anatomical images. 
Table 1. Activity evoked by stimulus 1 (all trials)

\begin{tabular}{|c|c|c|c|c|}
\hline \multirow[b]{2}{*}{ Brain area } & \multicolumn{3}{|c|}{$\begin{array}{l}\text { Experimental plus control } \\
\text { (STIM1 vs resting baseline) }\end{array}$} & \multirow[b]{2}{*}{$t$ value } \\
\hline & $x$ & $y$ & $Z$ & \\
\hline Left SI/PPC & -39 & -40 & 40 & 4.91 \\
\hline Right SI/PPC & 50 & -31 & 35 & 7.58 \\
\hline Left SII & -54 & -25 & 24 & 7.36 \\
\hline Right SII & 57 & -22 & 19 & 4.30 \\
\hline Left alC & -39 & 2 & 7 & 10.07 \\
\hline Right alC & 30 & 5 & 13 & 7.58 \\
\hline Left ACC (BA 24) & -4 & 11 & 28 & 2.10 \\
\hline Right ACC (BA 24) & 6 & 8 & 34 & 2.49 \\
\hline
\end{tabular}

To assess memory-related processes within areas activated by noxious stimuli, a stimulus-specific ROI mask was established based on the responses evoked by the first noxious heat stimulus (STIM1; data averaged across all trials and all subjects, including both control trials and experimental memory trials). The search for stimulus-related activation was narrowed to include only the cerebral regions commonly reported in studies of experimental pain (Apkarian et al., 2005); thus, the threshold $t$ value for the search volume of common pain regions is 4.07 (randomeffects GLM), which is equivalent to $p=0.05$, corrected for multiple comparisons within the 2074 voxels of the common pain network. Coordinates are given in Talairach space (Talairach and Tournoux, 1988). Lateral ( $x$ ), anterior $(y)$, and superior (z) stereotaxic coordinates (in millimeters) are relative to midline, anterior commissure, and commissural line, respectively (positive values are right, anterior, and superior). Left-side activation is contralateral to the presentation of noxious stimuli. BA, Brodmann area; $t$ values in bold correspond to a $p$ value of $\leq 0.05$.

The major thrust of this study was to test for possible evidence of short-term memory-related processes within regions of the brain that are initially responsive to the sensory-discriminative aspects of pain. Thus, the first step in our analysis process was to establish an appropriate region of interest (ROI) for this directed search. To define this pain-processing ROI, we performed a contrast between activation evoked by the first noxious stimulus (STIM1) delivered in each trial and the baseline period that separated the trials (Fig. 1, the final period of each trial). Both experimental and control trials were included in this analysis to ensure a robust stimulus-evoked response and hence a relatively inclusive ROI; activation evoked during the second noxious stimulus was not used to form this stimulus-related mask, because responses during this period were more likely to include memory- and/or discrimination-related processes, in addition to those directly related to pain. To minimize extraneous activation during this period, which might be less likely to have been directly related to the noxious stimuli, this search for stimulusrelated activation was restricted to regions of the brain that are generally associated with pain processing (Apkarian et al., 2005): bilateral regions of the postcentral gyrus, superior parietal lobule, cingulate region, insula, inferior and middle frontal gyri (search volume, $240-273 \mathrm{~mm}^{3}$ ). Activation evoked by the noxious stimuli, as revealed by this contrast, was thus used as an objective definition of the pain-processing ROI (Table 1). Boundaries for this ROI were automatically determined by the strict statistical criterion of $p=0.05(t=4.07)$, random-effect general linear model (GLM), Bonferroni-corrected for multiple comparisons [using stat-threshold (http://www.math.mcgill.ca/keith/fmristat/)] within the cerebral volume of pain responsiveness established previously (Apkarian et al., 2005). Thus, using these criteria, the pain ROI used in our initial search for short-term memory associated with noxious stimuli included bilateral regions of the SI/posterior parietal cortex (SI/PPC), SII, and IC. Although areas of activation were also observed bilaterally in the ACC, a region frequently associated with pain affect, these failed to reach the threshold of significance established for our ROI, perhaps reflecting the strong emphasis placed on subjects' attention toward sensorydiscriminative aspects of the noxious stimuli.

A secondary objective was to identify brain regions involved in memory and discrimination, outside the network of pain processing, for which we performed a whole-brain random-effect analysis, with results described for activation reaching a significance of $p=0.05(t=4.7)$, random-effect GLM, corrected for multiple comparisons within the more inclusive, unmasked whole-brain volume, as described above.

For confirmation of activation sites in an individual subject, a fixedeffect GLM was computed, and statistical thresholds were established at $p=0.05$, corrected for the appropriate search volume, as described above. Coordinates of loci of activation are given in Talairach space (Talairach and Tournoux, 1988).
All statistical contrasts conducted for the group analyses were performed using a random-effect GLM (Friston et al., 1999), and results were corrected for multiple comparisons for the specific search volume, as indicated above. Each distinct period within a trial that contained a stimulus, behavioral, or response event was declared as a regressor; the instruction cue period, ISIs, and baseline and post-response periods were, likewise, defined as regressors, and each event was further subcategorized according to trial type (experimental or control). Contrasts for stimulus-related or memory-related effects compared separately for each of the conditions (experimental and control) the appropriate stimulus or interstimulus "memory" regressor to the baseline regressor, which occurred at the end of the trial; all other regressors that are not part of the contrast are automatically entered as covariates of no interest in the analysis. This approach thus minimizes the influence of other events that are not directly compared with the GLM. Contrasts for memory-specific effects compared experimental and control trials for the appropriate regressor.

In summary, the strategy of the analysis was conceived to avoid any potential confounds of long-term memory and learning. All analyses are averaged across the full experimental session and thus do not represent any learning-related changes in perception that might occur across the different experimental runs within a session. Likewise, time course data are averaged across all trials and all sessions and thus do not show any trial-to-trial changes in perception.

\section{Results}

\section{Behavioral results}

During both memory and control tasks, all subjects described the noxious heat stimuli as painful but tolerable. Likewise, the reaction time to detect the offset of the noxious stimuli did not differ significantly between the experimental memory $(3134 \mathrm{~ms})$ and control (3143 ms) conditions $(F=0.011 ; p=0.916)$ nor between the first $(3153 \mathrm{~ms})$ and second $(3123 \mathrm{~ms})$ presentations of the stimulus in each trial $(F=0.008 ; p=0.927)$. These behavioral results confirm that the subjects' perceptions were consistent with the experience of painful heat and that the subjects engaged in similar levels of attention directed toward each of the two noxious stimuli presented within the two tasks. The subjects' performance in making spatial-intensity discriminations between the pairs of stimuli in each trial was uniformly low (mean, $59 \%$; SE, 9\%) and did not vary in a reliable manner with the variable size of $\Delta T$. Our failure to show a correlation between performance and $\Delta T$ reflects the difficulty of the psychophysical task, which was designed to minimize the subjects' tendency to categorize the intensity of the stimuli. In addition, comparisons between two thermodes (which may have slightly different stimulation characteristics) as well as comparisons between two different stimulus locations (which may, likewise, have different perceptual characteristics) might also have contributed to the inherent difficulty of the task. However, this situation did not appear to disturb subjects or interfere with their concentration on the task, because there was no presentation of feedback, which could have created a discrepancy between the stimulus characteristics and the subjects' perception of those characteristics.

\section{Stimulus-related activity}

The pain ROI described in Materials and Methods was defined using the most robust comparison available in this study: the initial noxious stimulus (STIM1) versus baseline, averaged across all trials, including both experimental (memory task) and control. Separate analyses of experimental trials and control trials, comparing STIM1 versus baseline, likewise demonstrated significant (or near-significant) activation in most of these regions within the pain ROI (Table 2), despite the reduced number of noxious stimuli included within each analysis. A direct compar- 
Table 2. Stimulus 1 (STIM1) activity: experimental versus control trials

\begin{tabular}{|c|c|c|c|c|c|c|c|c|c|c|c|c|}
\hline \multirow[b]{2}{*}{ Brain area } & \multicolumn{3}{|c|}{$\begin{array}{l}\text { Experimental STIM1 vs } \\
\text { resting baseline }\end{array}$} & \multirow[b]{2}{*}{$t$ value } & \multicolumn{3}{|c|}{ Control STIM1 vs resting baseline } & \multirow[b]{2}{*}{$t$ value } & \multicolumn{3}{|c|}{ Experimental vs control STIM1 } & \multirow[b]{2}{*}{$t$ value } \\
\hline & $x$ & $y$ & $Z$ & & $x$ & $y$ & $Z$ & & $x$ & $y$ & $Z$ & \\
\hline Left SI/PPC & -39 & -40 & 36 & 3.37 & -40 & -38 & 35 & 4.39 & -42 & -34 & 34 & 1.22 \\
\hline Right SI/PPC & 51 & -31 & 34 & 4.92 & 54 & -33 & 31 & 6.44 & 54 & -35 & 31 & 2.38 \\
\hline Left SII & -57 & -25 & 23 & 5.88 & -54 & -25 & 23 & 6.52 & -58 & -26 & 22 & 2.85 \\
\hline Right SII & 52 & -22 & 19 & 3.79 & 57 & -22 & 20 & 6.85 & 51 & -22 & 19 & 0.50 \\
\hline Left alC & -38 & 3 & 10 & 6.80 & -40 & 1 & 6 & 6.53 & -37 & 11 & 11 & 5.10 \\
\hline Right alC & 33 & 5 & 10 & 6.28 & 32 & 8 & 13 & 5.71 & 35 & 8 & 10 & 0.84 \\
\hline
\end{tabular}

Activity levels during the first and second (see Table 3) noxious thermal stimulus periods were first analyzed separately for experimental (delayed-discrimination, memory task) and control trials and then directly compared between these two conditions. A $t$ statistic of 3.70 (random-effects GLM) is equivalent to $p=0.05$, corrected for multiple comparisons within the stimulus-specific mask of pain-related activation demonstrated in the current study (467 voxels); $t$ values in bold correspond to a $p$ value of $\leq 0.05$. Within the network of brain areas normally responsive to pain (Apkarian et al., 2005), activation during the presentation of the first stimulus was generally robust; however, direct comparisons of these responses between experimental (memory) and control trials revealed no significant activation specific to the memory task, with the exception of the contralateral IC, which was significantly more active during the presentation of stimulus 1 in the experimental trials, in which subjects were required to encode and compare the sensory features of the stimuli.

Table 3. Stimulus 2 (STIM2) activity: experimental versus control trials

\begin{tabular}{|c|c|c|c|c|c|c|c|c|c|c|c|c|}
\hline \multirow[b]{2}{*}{ Brain area } & \multicolumn{3}{|c|}{$\begin{array}{l}\text { Experimental STIM2 vs } \\
\text { resting baseline }\end{array}$} & \multirow[b]{2}{*}{$t$ value } & \multicolumn{3}{|c|}{ Control STIM2 vs resting baseline } & \multirow[b]{2}{*}{$t$ value } & \multicolumn{3}{|c|}{ Experimental vs control STIM2 } & \multirow[b]{2}{*}{$t$ value } \\
\hline & $x$ & $y$ & $Z$ & & $x$ & $y$ & $Z$ & & $x$ & $y$ & $Z$ & \\
\hline Left SI/PPC & -40 & -39 & 36 & 6.19 & -33 & -36 & 35 & 5.63 & -51 & -32 & 34 & 2.65 \\
\hline Right SI/PPC & 43 & -31 & 40 & 9.31 & 49 & -35 & 35 & 9.78 & 43 & -33 & 34 & 2.55 \\
\hline Left SII & -54 & -25 & 22 & 7.31 & -55 & -25 & 21 & 6.99 & -57 & -25 & 21 & 3.01 \\
\hline Right SII & 53 & -25 & 21 & 5.45 & 57 & -25 & 20 & 6.60 & 54 & -22 & 21 & 1.03 \\
\hline Left alC & -42 & -1 & 7 & 11.57 & -33 & 11 & 12 & 11.47 & -42 & 8 & 7 & 5.43 \\
\hline Right alC & 33 & -1 & 10 & 13.32 & 33 & 14 & 10 & 9.34 & 36 & 8 & 13 & 3.07 \\
\hline
\end{tabular}

Activity levels during the second noxious thermal stimulus period were analyzed as described in Table 2. Within the network of brain areas normally responsive to pain (Apkarian et al., 2005), activation during the presentation of the second stimulus was especially robust. However, as indicated for STIM1 responses in Table 2, direct comparison of experimental (memory) and control trials revealed no significant activation specific to the memory task, with the exception of the contralateral IC.

Table 4. Memory-specific activity in individual subjects during the delay (ISI) within the pain ROI

\begin{tabular}{lllll}
\hline Subject & Brain area & $x$ & $y$ & $z$ \\
\hline 1 & SI & -33 & -36 & 46 \\
2 & PPC & -33 & -50 & 46 \\
3 & SI & -40 & -34 & 46 \\
4 & SI/IPL & -45 & -37 & 31 \\
5 & SI/IPL & -48 & -40 & 34 \\
6 & SI & -31 & -43 & 53 \\
7 & IPL & -60 & -32 & 31 \\
8 & SI & -34 & -46 & 59 \\
\hline
\end{tabular}

Data are memory-specific activity during the delay period (ISI) obtained from the direct subtraction of control from experimental (delayed-discrimination, memory task) trials in individual subjects. The anatomical determination of the activation sites was made from a careful examination of the data associated with each subject, relative to his/her own specific parietal anatomy. IPL, Inferior parietal lobule.

ison of the experimental and control trials for activation during the STIM1 period, demonstrated that responses to the noxious stimulation during these two conditions was remarkably similar, with the single exception of the contralateral (left) IC, which was significantly more responsive to noxious stimuli presented during the experimental memory condition (Table 2). Analyses of data recorded during presentation of the second noxious stimulus (STIM2) of each trial demonstrated somewhat stronger levels of activation within the pain ROI during both experimental and control conditions (Table 3) compared with that observed for STIM1, and, as was observed during STIM1, stimulus-related responses within the pain ROI during STIM2 were very similar for the two conditions, except for the left contralateral anterior IC (aIC), where the activation during experimental trials was significantly stronger than those of the control condition.

For stimulus-related activity detailed in Tables 2 and 3 , as well as for memory-related activation described below, we have classified parietal regions of activation as "SI/PPC," to reflect the spatial variability of activation sites observed in this group of subjects. In assessing the actual location of stimulus-related acti- vation sites in parietal cortex, functional data associated with each subject were examined relative to his own specific parietal landmarks using the high-resolution anatomical MRI recorded for each subject (Table 4). Four of the eight subjects exhibited detectable activation within the postcentral gyrus (SI), parietal activation from two subjects appeared at the border between SI and the inferior parietal lobe, and activity from the remaining two subjects was either posterior or inferior to SI proper. Thus, although the majority of subjects exhibited stimulus- and memoryrelated activation in the general region of SI, we indicate this more inclusively as SI/PPC.

Together, the stimulus-related activation, within the generally acknowledged pain network, is consistent with the subjects' description of the noxious stimuli as painful. These data also serve as a validation for the perceptual demands of the control trials, in that subjects appear to have perceived and processed the noxious stimuli similarly in both tasks, independent of differences in cognitive strategies evoked by task-specific instruction cues that preceded each trial.

\section{Memory-related activity}

To elucidate pain-processing areas potentially involved in the short-term memory of painful sensations, activity within the pain ROI, recorded during the ISI, was analyzed for both the experimental memory trials and the control trials. Figure 2 and Table 5 illustrate the highly significant activation (averaged across all subjects) that was observed during both tasks during the ISI compared with the resting baseline activity. All regions within the pain ROI, which had shown significant STIM1-related activation 
(Table 2), demonstrated even stronger levels of activity after those stimuli were terminated, during the delay that preceded the second stimulus (Table 5). However, activation that was specific to the memory requirements of the experimental trials was observed in only a subset of these regions. A direct contrast of experimental and control trials for ISI activation revealed significant memoryspecific activation in contralateral SI/PPC and in bilateral aIC (Fig. 2); memoryspecific activity in ipsilateral SI/PPC showed a trend toward significance (Table 5) $(t=3.45$ compared with the threshold of $t=3.7)$ in this comparison of experimental and control trials. Memoryspecific activation was also observed in a second region within the ipsilateral aIC at its border with the inferior frontal gyrus, an area that had been defined within the pain ROI (Table 5). In contrast, we could not detect even a trend toward memoryrelated modulation of activity in SII (Fig. 2 , Table 5$)$ ( $t \geq 1.59 ; p=1$, corrected for multiple comparisons).

Comparison of the time-course data, averaged across all subjects for the two tasks, illustrates the significantly enhanced BOLD signal recorded in the regions of activation within SI/PPC and aIC during the memory trials, compared with that of the control trials, beginning with the onset of the interstimulus memory period following the initial noxious stimulus (Fig. 3 ). Although activity recorded during the control trials dropped to near-baseline levels before the second noxious stimulus, activation during memory trials remained at an enhanced level in all four of these brain regions until the subjects made their final discrimination response.

\section{Discrimination-related activity}

The delayed discrimination task, used in the experimental trials, required subjects to remember sensory aspects of the first noxious stimulus for the duration of the interstimulus delay, and thus activity during the delay is directly associated with short-term memory of the pain stimulus, as we have presented above. Also, inherent to this task is an active comparison and ultimate discrimination of the two stimuli, a process that begins with the onset of STIM2 (Oshiro et al., 2006). One would hypothesize that any potential discrimination-related activity that might occur within the pain-processing areas would be revealed by a comparison of responses recorded during experimental and control trials for activation that was specific to the STIM2 period. The possibility of this additional discriminative process, during the presentation of STIM2, led to our exclusion of STIM2 activity from the formulation of the pain ROI, in which we wanted to maximize stimulus-related (as opposed to cognitive) activation. A comparison of the results illustrated in Tables 2 and 3 support the hypothesis that some additional process, or modulation of activity, may occur during STIM2, because activation appears stronger

\section{Activity during the Delay Period (InterStimulus Interval)}

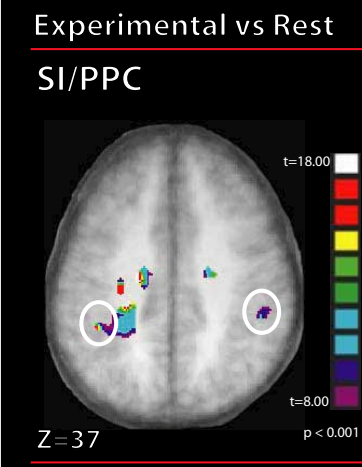

Control vs Rest

SI/PPC

Experimental minus Control

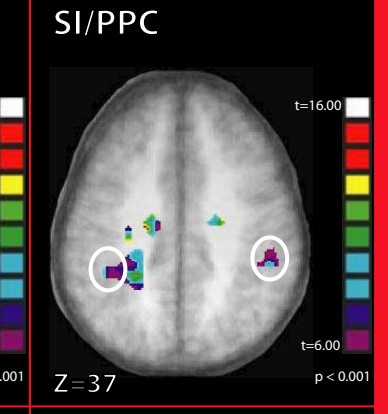

SI/PPC

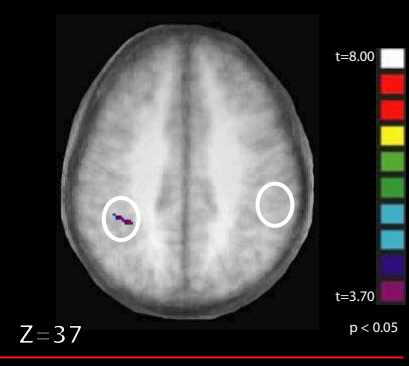

$\mathrm{Z}=37$
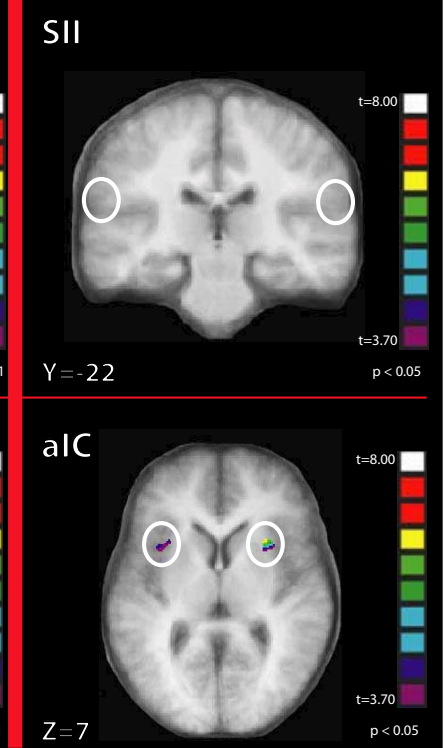

a

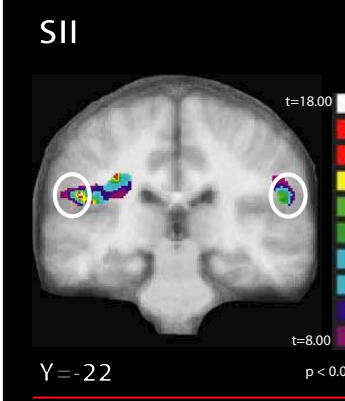

Figure 2. Cortical regions significantly activated during the ISI between the pairs of stimuli in both the memory and control trials and memory-specific activation observed within pain-related sites. Delay-related activation, assessed by contrasting the $B O L D$ responses associated with the delay and the baseline, was similar for both experimental and control trials in bilateral SI/PPC, (illustrations are focused on BOLD responses exceeding a significance level of $p<0.001$, corrected for multiple

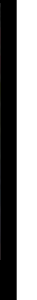

alc

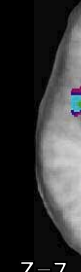

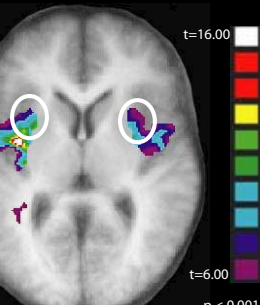

SII
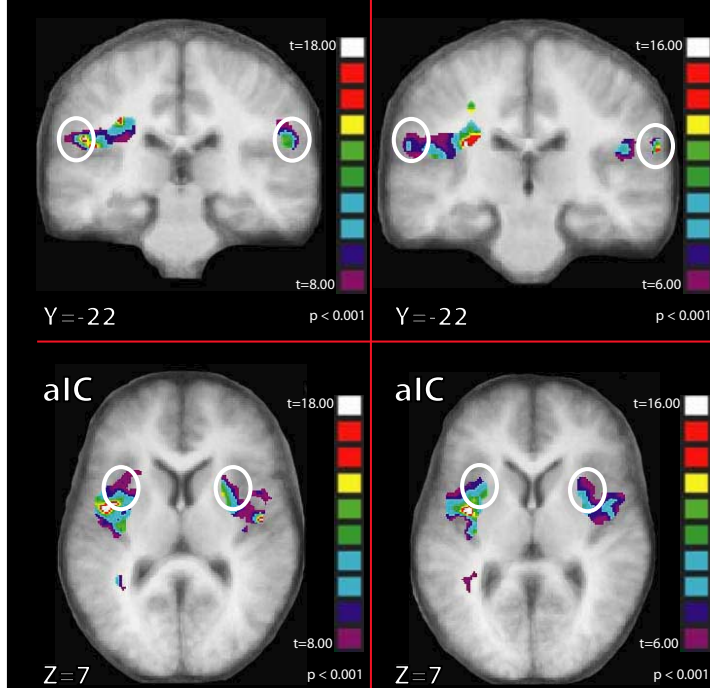

multiple comparisons). No trends toward memory-specific activation were seen within SII.

during STIM2 than during STIM1 when analyzed relative to the resting baseline activity. A direct comparison of activation levels observed during the two stimulation periods is presented in Table 6, confirming in both experimental and control trials the significantly stronger activation during STIM2 in most regions within the pain ROI (activation during control trials within right SI/PPC and right SII shows strong trends but fails to reach the significance threshold of $t=3.7)$. However, this increased activity in the pain ROI during STIM2 is not convincingly specific to the discrimination process itself, because the effect was also observed during control trials, when subjects were not required to discriminate the pairs of stimuli. Thus, no significant differences between experimental memory/discrimination trials and control trials were observed in the enhanced STIM2 activity within the pain ROI.

A global search of the entire brain, for evidence of discrimination-related activity that might occur outside the network of pain-responsive regions, revealed activation within the left ventrolateral prefrontal cortex (VLPFC) that was significantly stronger during the STIM2 period compared with that of the 
Table 5. Memory-specific activity during the delay (ISI) within the pain ROI

\begin{tabular}{|c|c|c|c|c|c|c|c|c|c|c|c|c|}
\hline \multirow[b]{2}{*}{ Brain area } & \multicolumn{3}{|c|}{$\begin{array}{l}\text { Experimental delay vs resting } \\
\text { baseline }\end{array}$} & \multirow[b]{2}{*}{$t$ value } & \multicolumn{3}{|c|}{ Control delay vs resting baseline } & \multirow[b]{2}{*}{$t$ value } & \multicolumn{3}{|c|}{$\begin{array}{l}\text { Memory-specific (experimental } \\
\text { vs control) }\end{array}$} & \multirow[b]{2}{*}{$t$ value } \\
\hline & $x$ & $y$ & $z$ & & $x$ & $y$ & $z$ & & $x$ & $y$ & $z$ & \\
\hline Left SI/PPC & -39 & -40 & 37 & 17.78 & -39 & -40 & 37 & 8.44 & -39 & -40 & 37 & 4.87 \\
\hline Right SI/PPC & 45 & -34 & 37 & 10.65 & 48 & -35 & 34 & 9.80 & 48 & -35 & 37 & 3.45 \\
\hline Left SII & -53 & -25 & 23 & 9.47 & -57 & -22 & 19 & 8.95 & -54 & -23 & 20 & 0.29 \\
\hline Right SII & 54 & -26 & 19 & 13.36 & 61 & -22 & 19 & 15.17 & 59 & -24 & 20 & 1.59 \\
\hline Left alC & -33 & 11 & 12 & 9.25 & -30 & 11 & 7 & 8.31 & -30 & 11 & 13 & 4.34 \\
\hline Right alC & 27 & 13 & 7 & 9.66 & 31 & 12 & 5 & 7.06 & 27 & 14 & 7 & 6.85 \\
\hline Right alC/IFG & 52 & -1 & 16 & 13.39 & 52 & -1 & 18 & 7.58 & 51 & 2 & 19 & 4.80 \\
\hline
\end{tabular}

Activity during the delay period (ISI) was first analyzed separately for experimental (delayed-discrimination, memory task) and control trials and then directly compared between the two conditions. Analyses are restricted to the ROI defined by stimulus-specific pain-related activity evoked by stimulus 1, with significance thresholds as described in Tables 2 and 3 . Memory-specific activation during the delay period was observed in several regions of the pain-responsive ROl; however, the perisylvian regions (including SII), which were robustly active during presentation of the two stimuli, did not show significant memory-specific activation during the interstimulus delay between the two stimuli. IFG, Inferior frontal gyrus. $t$ values in bold correspond to a $p$ value of $\leq 0.05$.

Contralateral SI/PPC

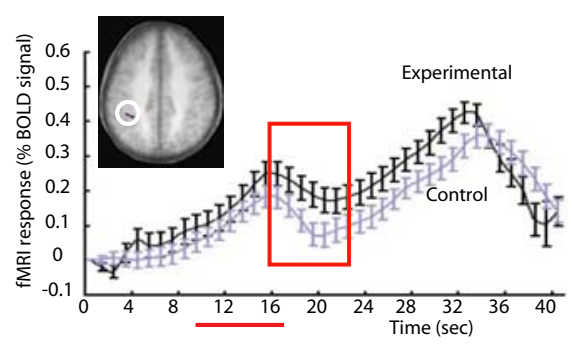

Contralateral Anterior Insular Cortex

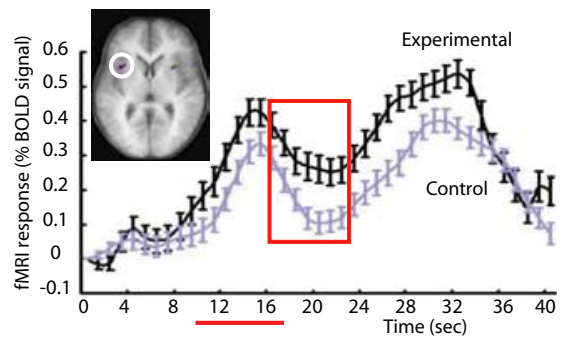

Ipsilateral SI/PPC

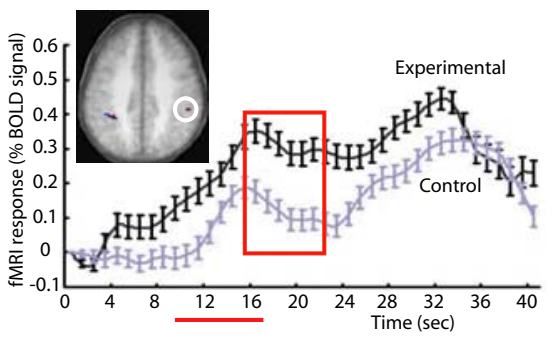

Ipsilateral Anterior Insular Cortex

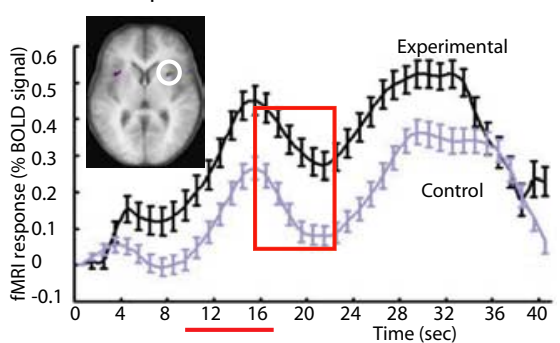

Figure 3. Mean time course of the memory-specific percentage BOLD signal from the group average observed during the course of experimental and control trials, synchronized on the start of each trial. In all cases illustrated, the BOLD signal increase observed during the ISI is significantly higher during experimental memory trials compared with that observed during the same interval of the control trials. Time 0 is the onset of the trial; the red box illustrates the expected time period of peak BOLD responses associated with the ISI, after taking into consideration the delay of the hemodynamic response function.

STIM1 period. This effect was extremely robust for experimental trials (Table 6) $(t=11.49)$ and significantly greater than that observed during control trials (Table 6) $(t=5.47)$, suggesting a process specific to the task of discriminating the two stimuli.

\section{Global analyses}

To test for the occurrence of memory- and/or discriminationspecific activation outside of the regions initially responsive to the noxious stimulation, we performed a GLM analysis of the entire brain comparing activation observed during experimental trials with that observed during the control trials. Table 7 tabulates these results using a conservative threshold appropriate for this nondirected search $(t=4.7$, corrected for multiple comparisons across the entire brain volume). In the left thalamus and right aIC/inferior frontal gyrus, significant memory-specific activation was observed during the delay between noxious stimuli but not during the presentation of those stimuli. In the ACC, we had observed a slight trend toward pain-related activation during presentation of the first stimulus (Table 1$)(t<2.5)$, but no significant differences were observed in the ACC between exper- imental and control trials, except in the left ACC during presentation of the second stimulus (Table 7$)(t=8.82)$. In the right pre-supplementary motor area (preSMA) and bilateral VLPFC, activation was significantly greater in the experimental trials, compared with the control trials, during the delay between the two noxious stimuli; this enhancement of task-related activity continued throughout presentation of the second noxious stimulus, although neither of these regions had responded significantly to the initial presentation of the noxious stimuli.

\section{Discussion}

The present study investigated the shortterm memory of pain sensation in the human brain. Within the cerebral network of regions known to be responsive to noxious stimulation, we found a significant memory-specific enhancement of fMRI activity in contralateral SI/PPC and bilateral aIC during the intertrial interval of a nociceptive spatial/intensity discrimination task. These parietal and insular regions of memory-specific activation were also responsive to the noxious stimuli presented during both experimental and control tasks and have been shown previously to be associated with sensory-discriminative aspects of noxious and/or innocuous thermal perception. Although no other studies have examined the specific somatosensory processes involved in the short-term memory of pain, several have described neuronal correlates of short-term memory for innocuous cutaneous stimuli, but their results have been conflicting.

Consistent with our findings are those of a single-unit recording study, which demonstrated significant activation in monkey SI during the ISI of a delayed texture discrimination task (Zhou and Fuster, 1996; Bodner et al., 2005). It has also been shown in human subjects that transcranial magnetic stimulation (TMS) over SI during the ISI between two vibrotactile stimuli interfered with a delayed spatial discrimination (Harris et al., 2002), suggesting that human SI is a transient storage site for sensory aspects of innocuous vibrotactile stimuli. Likewise, in a recent fMRI study of normal human volunteers, Preuschhof et al. (2006) observed enhanced SI activation during a short delay between innocuous vibrotactile stimuli presented in a memory task. How- 
Table 6. Memory- and/or discrimination-specific activation during stimulus 2 (STIM2)

\begin{tabular}{|c|c|c|c|c|c|c|c|c|c|c|c|c|}
\hline \multirow[b]{2}{*}{ Brain area } & \multicolumn{3}{|c|}{$\begin{array}{l}\text { Experimental } \\
\text { (STIM2 vs STIM1) }\end{array}$} & \multirow[b]{2}{*}{$t$ value } & \multicolumn{3}{|c|}{ Control (STIM2 vs STIM1) } & \multirow[b]{2}{*}{$t$ value } & \multicolumn{3}{|c|}{$\begin{array}{l}\text { Experimental vs control } \\
\text { (STIM2 vs STIM1) }\end{array}$} & \multirow[b]{2}{*}{$t$ value } \\
\hline & $x$ & $y$ & $z$ & & $x$ & $y$ & $z$ & & $x$ & $y$ & $Z$ & \\
\hline \multicolumn{13}{|c|}{ Directed search within the pain $\mathrm{ROI}^{a}$} \\
\hline Right SI/PPC & 45 & -34 & 37 & 4.09 & 48 & -30 & 34 & 3.25 & 36 & -31 & 35 & 3.55 \\
\hline Left SII & -57 & -25 & 13 & 4.73 & -50 & -25 & 15 & 4.87 & -51 & -25 & 18 & 0.19 \\
\hline Right SII & 46 & -23 & 13 & 4.42 & 50 & -25 & 19 & 3.23 & 45 & -22 & 13 & 0.88 \\
\hline Left alC & -42 & 8 & 7 & 5.62 & -33 & -1 & 16 & 9.04 & -33 & 11 & 1 & 2.13 \\
\hline Right alC & 27 & 9 & 12 & 5.74 & 39 & 2 & 17 & 4.82 & 36 & 8 & 13 & 2.50 \\
\hline \multicolumn{13}{|c|}{ Global search of entire brain ${ }^{b}$} \\
\hline Left VLPFC & -36 & 47 & 10 & 11.49 & -35 & 47 & 10 & 2.32 & -30 & 44 & 13 & 5.47 \\
\hline
\end{tabular}

${ }^{a}$ Data from the period of the second stimulus are analyzed and compared with those of the first stimulus (STIM1) for experimental and control trials. During stimulus 1 of experimental trials, subjects attended to sensory qualities of the noxious stimulation and registered those features in memory to make a subsequent discrimination; during stimulus 2 of the experimental trials, subjects likewise attended to the noxious stimuli and initiated the discrimination process, comparing the current sensation with that remembered from the first stimulation period. Activity within stimulus-related regions was, in general, significantly greater during the second stimulation period compared with that observed during the first stimulation period. However, this enhanced responsiveness to stimulus 2 within stimulus-related regions is not convincingly specific to the discrimination process itself, because the effect was also observed during control trials, when subjects were not required to discriminate the pairs of stimuli. Analyses are restricted to the previously described stimulus-specific ROI, with a significance threshold of $t=3.70, p=0.05$, as described in Tables 2 and $3 . t$ values in bold correspond to a $p$ value of $\leq 0.05$.

${ }^{b}$ Data from the period of the second stimulus were also analyzed and compared with those of the first stimulus for experimental and control trials after performing a global search of the entire brain. The threshold for significance was $t=$ $4.70, p=0.05$.

Table 7. Global-search analysis of the entire brain: additional activation areas specific to memory and/or discrimination

\begin{tabular}{|c|c|c|c|c|c|c|c|c|c|c|c|c|}
\hline \multirow[b]{3}{*}{ Brain area } & \multicolumn{12}{|c|}{ Experimental vs control tasks } \\
\hline & \multicolumn{3}{|l|}{ STIM1 } & \multirow[b]{2}{*}{$t$ value } & \multicolumn{3}{|c|}{ Delay (ISI) } & \multirow[b]{2}{*}{$t$ value } & \multicolumn{3}{|l|}{ STIM2 } & \multirow[b]{2}{*}{$t$ value } \\
\hline & $x$ & $y$ & $Z$ & & $x$ & $y$ & $Z$ & & $x$ & $y$ & $Z$ & \\
\hline Left thalamus & -16 & -20 & 10 & 0.30 & -15 & -19 & 10 & 6.32 & -13 & -19 & 0 & 1.29 \\
\hline Right alC/IFG & 39 & 17 & 7 & 3.78 & 39 & 14 & 4 & 4.92 & 36 & 20 & 4 & 3.42 \\
\hline Left ACC & -9 & 14 & 37 & 2.49 & -9 & 35 & 19 & 3.87 & -6 & 17 & 31 & 8.82 \\
\hline Right ACC & 8 & 2 & 32 & 2.09 & 7 & 23 & 34 & 3.72 & 9 & 26 & 24 & 2.82 \\
\hline Right pre-SMA & 12 & -4 & 49 & 3.04 & 0 & 11 & 49 & 5.83 & 9 & 8 & 46 & 7.93 \\
\hline Left VLPFC & -26 & 37 & 4 & 0.25 & -33 & 23 & 31 & 5.91 & -36 & 38 & 10 & 10.39 \\
\hline Right VLPFC & 39 & 41 & 4 & 2.04 & 45 & 41 & 13 & 5.98 & 27 & 47 & 4 & 6.92 \\
\hline
\end{tabular}

In addition to activation areas observed during directed searches within the stimulus-related ROI, a number of other regions were noted with significant activity specific to encoding of sensory features of stimulus 1 (STIM1 period, experimental vs control trials), short-term memory (delay period, experimental vs control trials), or discrimination [stimulus 2 (STIM2) period, experimental vs control trials] of the noxious thermal stimuli [random-effects GLM analyses, global search of entire brain volume (threshold of significance, $t=4.7 ; p=0.05$, corrected for multiple comparisons) ]. Stereotaxic coordinates are given in Talairach space (Talairach and Tournoux, 1988). IFG, Inferior frontal gyrus. $t$ values in bold correspond to a $p$ value of $\leq 0.05$.

ever, results of these studies remain inconclusive: ISI "memoryrelated" activity in monkey SI did not actually encode information specific to the remembered target stimulus (Bodner et al., 2005); TMS-related interference in a discrimination task may be related to generalized corticocortical effects of the stimulation, rather than to a direct, specific effect on SI; and Preuschhof et al. (2006) only observed the enhanced activation in SI during the first $100 \mathrm{~ms}$ of the ISI memory period, not during longer $4 \mathrm{~s}$ ISIs. In our present study, the limited sensitivity of the fMRI methods and anatomical variability of our subjects do not permit us to restrict our conclusions to SI proper or to distinguish between possible stimulus-specific differences in either the intensity or the location of memory-related activation. Future studies with greater sensitivity may be able to address this issue.

The memory-related responses that we observed extending into the PPC (left PPC, contralateral to the noxious stimulation: $t=4.87, p<0.05$; right ipsilateral PPC: $t=3.45 ; p=0.13)$ are consistent with the recognized role of these structures in modulating both noxious and innocuous input by cognitive variables, including attention and memory (Apkarian et al., 2005). The right PPC has been implicated in the process of orienting attention toward somatosensory stimuli and appears to be responsive to cutaneous stimuli delivered to either side of the body (Coghill et al., 2001). Here, activation of the PPC may reflect the critical attention toward the spatial information required to perform the memory task.

Our observation of robust ipsilateral sites of stimulus-related activation within SI/PPC (Tables 1-3) may appear surprising, considering traditional views regarding the predominant representation of a contralateral body topography within the primary somatosensory cortex. However, converging evidence from lesion studies, intracranial recordings, and fMRI results is consistent with activation of SI ipsilateral to the presentation of an innocuous cutaneous stimulus to the hand (for review, see Sutherland, 2006). Likewise, several recent fMRI studies using experimental noxious stimuli also suggest an ipsilateral or bilateral activation of SI associated with painful stimulation of the hand (Bornhovd et al., 2002; Bingel et al., 2004).

The absence, in our data, of any activity indicating a role for SII in pain memory contrasts with results of Romo and colleagues (Salinas et al., 2000), who reported memory-related activity in monkey SII during the interval between two innocuous vibrotactile stimuli; however, those monkeys were trained to discriminate the frequency of vibrotactile stimuli presented to a single spot of skin, whereas our subjects were required to remember the spatial location of different noxious pain stimuli, a task we designed to engage more specifically the functional capacity associated with the fine somatotopic organization found in SI. In agreement with our data are the results of Preuschhof et al. (2006), which, likewise, show no indication in human subjects of SII involvement in maintaining a vibrotactile memory trace.

Analysis of the memory delay period also revealed significant activation in anterior insula bilaterally, but with a considerably stronger response on the ipsilateral right side (Table 5). The aIC is part of the limbic system and is sometimes implicated in the perception and processing of affective aspects of pain (Bushnell et 
al., 1999; Brooks et al., 2005) and in the recognition of acute pain in others (Singer et al., 2004; Jackson et al., 2005). However, it has also been associated with sensory-discriminative encoding of innocuous cutaneous stimuli, such as the perception of coolness (Craig et al., 2000) or warmth (Olausson et al., 2005), during tasks essentially devoid of negative affect. Our paradigm does not allow us to distinguish whether the sensory or the affective aspects of the remembered painful stimulus mediate the memoryspecific activation of aIC; however, memory of the possible differences in affective aspects of the heat-pain stimuli would not have provided sufficient information to perform the spatialdiscrimination task. Regardless, the aIC is well situated to participate in cognitive and memory-related aspects of pain perception, considering its direct anatomical connections with the prefrontal cortex (Apkarian et al., 2005). This interpretation is consistent with the findings of Downar et al. (2000, 2002), which demonstrated that the right aIC is part of a cortical network subserving cognitive processes such as attention and detection of salient sensory (visual, auditory, and tactile) stimuli. In addition, anatomical studies in the macaque have linked aIC to short-term memory processes and have suggested that the aIC relays sensory information from SI and SII to the frontal cortex for short-term retention (Mesulam and Mufson, 1982) and to the temporal lobe for long-term encoding of tactile information (Friedman et al., 1986; Burton and Sinclair, 2000).

In addition to memory-related activation observed within the network of areas activated by the initial noxious stimulus, a global search of the entire brain revealed other areas that that were not initially responsive to the noxious stimuli but that nevertheless appear to participate in memory and discrimination processes during later stages of the experimental trials. A comparison of experimental and control trials revealed significant activation within bilateral mid-VLPFC and right pre-SMA that began after the termination of the initial stimulus and continued throughout the ISI and presentation of the second stimulus. This memoryand discrimination-specific activation is consistent with many previous studies demonstrating a role for the frontal lobes in working memory for innocuous cutaneous stimuli (Romo et al., 1999; Romo and Salinas, 2003), verbal material (Owen et al., 2005), and more generally in the active comparison and retrieval of information in both short- and long-term memory (Petrides, 2005). Activation in pre-SMA has been observed in vibrotactile short-term memory tasks in both monkey single-unit recordings (Romo and Salinas, 2003) and human fMRI studies (Preuschhof et al., 2006). In our present study, the pre-SMA activity during memory and discrimination periods of the trial only occurred on the right side contralateral to the hand that tapped the response key at the end of each trial. We had designed the experimental paradigm with pseudo-randomized response instructions that came only at the end of the trial, to minimize or delay any transfer of sensory memory to the planning of a specific motor response; however, because the final instruction was limited to only four possible combinations of stimulus location and appropriate response key, activation of this pre-SMA region is consistent with its proposed role in complex cognitive aspects of sensory-motor integration and the recall of motor memories (Romo and Salinas, 2003).

Our observation of the ISI-specific activation in VLPFC, an area acknowledged for its important role in memory, suggests that memory for spatial and intensity features of the noxious stimuli is also the most plausible explanation for activation seen during this period within the more posterior, sensory areas of the cortex. Alternatively, one might suggest that increased attention, arousal, and anticipation during the memory task could account for the modulation of activity observed between the presentation of the two noxious stimuli, because these cognitive factors can significantly modulate the BOLD response in the network of brain areas involved in pain perception (Porro et al., 2002; Koyama et al., 2005), including ACC (Mohr et al., 2005). However, in the present study, levels of attention, arousal, and anticipation were comparable in the memory and control tasks, as demonstrated by similarities in the subjects' responses to the detection of stimulus offset, which was common to the two tasks. Likewise, cerebral activation associated with the stimulus period was generally indistinguishable for the memory and control tasks. Thus, by subtracting the activation levels associated with the ISI in the control trials from the homologous period in the memory trials, we removed from the activation maps the contribution of the arousal induced by the previous stimulus as well as the expectation of the second stimulus. The only factors remaining after this subtraction should have been the necessity, during the memory task, to retain information related to the sensory features of the first noxious stimulus.

We did not observe any significant activation in ACC related to the short-term memory of the noxious stimuli during the ISI; the strongest trend toward activation was observed only at a $p$ value threshold of $0.5(t=3.87)$. The ACC is a limbic region involved in the processing of pain affect (Rainville et al., 1997; Singer et al., 2004; Apkarian et al., 2005). In our study, subjects perceived both the sensory (location and intensity) and affective (unpleasantness) aspects of the heat-pain stimuli and may have used the unpleasantness of the stimulus to encode its intensity. However, the absence of memory-specific activation in ACC suggests that subjects did not hold the affective aspect of the stimuli in memory, probably because that information would not have been sufficient to perform the delayed-discrimination paradigm.

We have shown that an increase in activation of the SI and aIC is associated with both the presentation of painful stimuli and the short ISI that separated painful stimuli in a spatial-intensity discrimination task. Previous studies have suggested a role for those structures in the sensory dimension of noxious and/or innocuous thermal stimuli (Bushnell et al., 1999; Hofbauer et al., 2001; Brooks et al., 2005; Olausson et al., 2005), and our data now indicate their importance in retaining a short-term memory trace of the sensory features required to perform a pain-discrimination task. Finally, the absence, in our study, of any trend toward memory-related activation within the ACC, an area previously suggested to underlie the memory of affect inherent to pain empathy (Singer et al., 2004), may suggest that the functional separation of sensory and affective aspects of pain perception appears to extend to the memory of pain as well.

\section{References}

Apkarian AV, Bushnell MC, Treede RD, Zubieta JK (2005) Human brain mechanisms of pain perception and regulation in health and disease. Eur J Pain 9:463-484.

Bingel U, Lorenz J, Glauche V, Knab R, Glascher J, Weiller C, Buchel C (2004) Somatotopic organization of human somatosensory cortices for pain: a single trial fMRI study. NeuroImage 23:224-232.

Bodner M, Shafi M, Zhou YD, Fuster JM (2005) Patterned firing of parietal cells in a haptic working memory task. Eur J Neurosci 21:2538-2546.

Bornhovd K, Quante M, Glauche V, Bromm B, Weiller C, Buchel C (2002) Painful stimuli evoke different stimulus-response functions in the amygdala, prefrontal, insula and somatosensory cortex: a single-trial fMRI study. Brain 125:1326-1336.

Brooks JC, Zambreanu L, Godinez A, Craig AD, Tracey I (2005) Somatotopic organisation of the human insula to painful heat studied with high resolution functional imaging. NeuroImage 27:201-209. 
Burton H, Sinclair RJ (2000) Attending to and remembering tactile stimuli: a review of brain imaging data and single-neuron responses. J Clin Neurophysiol 17:575-591.

Bushnell MC, Duncan GH, Hofbauer RK, Ha B, Chen JI, Carrier B (1999) Pain perception: is there a role for primary somatosensory cortex? Proc Natl Acad Sci USA 96:7705-7709.

Coghill RC, Gilron I, Iadarola MJ (2001) Hemispheric lateralization of somatosensory processing. J Neurophysiol 85:2602-2612.

Coghill RC, McHaffie JG, Yen YF (2003) Neural correlates of interindividual differences in the subjective experience of pain. Proc Natl Acad Sci USA 100:8538-8542.

Craig AD, Chen K, Bandy D, Reiman EM (2000) Thermosensory activation of insular cortex. Nat Neurosci 3:184-190.

Downar J, Crawley AP, Mikulis DJ, Davis KD (2000) A multimodal cortical network for the detection of changes in the sensory environment. Nat Neurosci 3:277-283.

Downar J, Crawley AP, Mikulis DJ, Davis KD (2002) A cortical network sensitive to stimulus salience in a neutral behavioral context across multiple sensory modalities. J Neurophysiol 87:615-620.

Friedman DP, Murray EA, O’Neill JB, Mishkin M (1986) Cortical connections of the somatosensory fields of the lateral sulcus of macaques: evidence for a corticolimbic pathway for touch. J Comp Neurol 252:323-347.

Friston KJ, Holmes AP, Worsley KJ (1999) How many subjects constitute a study? NeuroImage 10:1-5.

Harris JA, Miniussi C, Harris IM, Diamond ME (2002) Transient storage of a tactile memory trace in primary somatosensory cortex. J Neurosci 22:8720-8725.

Hofbauer RK, Rainville P, Duncan GH, Bushnell MC (2001) Cortical representation of the sensory dimension of pain. J Neurophysiol 86:402-411.

Jackson PL, Meltzoff AN, Decety J (2005) How do we perceive the pain of others? A window into the neural processes involved in empathy. NeuroImage 24:771-779.

Koyama T, McHaffie JG, Laurienti PJ, Coghill RC (2005) The subjective experience of pain: where expectations become reality. Proc Natl Acad Sci USA 102:12950-12955.

Mesulam MM, Mufson EJ (1982) Insula of the old world monkey. III: Efferent cortical output and comments on function. J Comp Neurol 212:38-52.

Mohr C, Binkofski F, Erdmann C, Buchel C, Helmchen C (2005) The anterior cingulate cortex contains distinct areas dissociating external from self-administered painful stimulation: a parametric fMRI study. Pain 114:347-357.

Morrison I, Lloyd D, di PG, Roberts N (2004) Vicarious responses to pain in anterior cingulate cortex: is empathy a multisensory issue? Cogn Affect Behav Neurosci 4:270-278.

Olausson H, Charron J, Marchand S, Villemure C, Strigo IA, Bushnell MC (2005) Feelings of warmth correlate with neural activity in right anterior insular cortex. Neurosci Lett 389:1-5.

Oshiro Y, Quevedo A, McHaffie J, Kraft R, Coghill R (2006) Brain mechanisms supporting intensity discrimination and memory of pain. J Pain $7: 20$.
Owen AM, McMillan KM, Laird AR, Bullmore E (2005) N-back working memory paradigm: a meta-analysis of normative functional neuroimaging studies. Hum Brain Mapp 25:46-59.

Pasternak T, Greenlee MW (2005) Working memory in primate sensory systems. Nat Rev Neurosci 6:97-107.

Petrides M (2005) Lateral prefrontal cortex: architectonic and functional organization. Philos Trans R Soc Lond B Biol Sci 360:781-795.

Porro CA, Cettolo V, Francescato MP, Baraldi P (1998) Temporal and intensity coding of pain in human cortex. J Neurophysiol 80:3312-3320.

Porro CA, Baraldi P, Pagnoni G, Serafini M, Facchin P, Maieron M, Nichelli P (2002) Does anticipation of pain affect cortical nociceptive systems? J Neurosci 22:3206-3214.

Preston SD, de Waal FB (2002) Empathy: its ultimate and proximate bases. Behav Brain Sci 25:1-20.

Preuschhof C, Heekeren HR, Taskin B, Schubert T, Villringer A (2006) Neural correlates of vibrotactile working memory in the human brain. J Neurosci 26:13231-13239.

Rainville P, Feine JS, Bushnell MC, Duncan GH (1992) A psychophysical comparison of sensory and affective responses to four modalities of experimental pain. Somatosens Mot Res 9:265-277.

Rainville P, Duncan GH, Price DD, Carrier B, Bushnell MC (1997) Pain affect encoded in human anterior cingulate but not somatosensory cortex. Science 277:968-971.

Rainville P, Doucet JC, Fortin MC, Duncan GH (2004) Rapid deterioration of pain sensory-discriminative information in short-term memory. Pain 110:605-615.

Romo R, Salinas E (2003) Flutter discrimination: neural codes, perception, memory and decision making. Nat Rev Neurosci 4:203-218.

Romo R, Brody CD, Hernandez A, Lemus L (1999) Neuronal correlates of parametric working memory in the prefrontal cortex. Nature 399:470-473.

Salinas E, Hernandez A, Zainos A, Romo R (2000) Periodicity and firing rate as candidate neural codes for the frequency of vibrotactile stimuli. J Neurosci 20:5503-5515.

Singer T, Seymour B, O’Doherty J, Kaube H, Dolan RJ, Frith CD (2004) Empathy for pain involves the affective but not sensory components of pain. Science 303:1157-1162.

Sutherland MT (2006) The hand and the ipsilateral primary somatosensory cortex. J Neurosci 26:8217-8218.

Taddio A, Goldbach M, Ipp M, Stevens B, Koren G (1995) Effect of neonatal circumcision on pain responses during vaccination in boys. Lancet 345:291-292.

Talairach J, Tournoux P (1988) Co-planar stereotaxic atlas of the human brain 3-dimensional proportional system: an approach to cerebral imaging. Stuttgart, Germany: Thieme Medical Publishers.

Wager TD, Rilling JK, Smith EE, Sokolik A, Casey KL, Davidson RJ, Kosslyn SM, Rose RM, Cohen JD (2004) Placebo-induced changes in FMRI in the anticipation and experience of pain. Science 303:1162-1167.

Woolf CJ, Salter MW (2000) Neuronal plasticity: increasing the gain in pain. Science 288:1765-1769.

Zhou YD, Fuster JM (1996) Mnemonic neuronal activity in somatosensory cortex. Proc Natl Acad Sci USA 93:10533-10537. 